\title{
Intermédialités
}

Histoire et théorie des arts, des lettres et des techniques

Intermediality

History and Theory of the Arts, Literature and Technologies

\section{Le tiers synesthète : espace d'accueil pour la création en langue des signes}

\section{Julie Chateauvert}

Numéro 27, printemps 2016

traduire

translating

URI : https://id.erudit.org/iderudit/1039816ar

DOI : https://doi.org/10.7202/1039816ar

Aller au sommaire du numéro

Éditeur(s)

Revue intermédialités (Presses de l’Université de Montréal)

ISSN

1920-3136 (numérique)

Découvrir la revue

Citer cet article

Chateauvert, J. (2016). Le tiers synesthète : espace d'accueil pour la création en langue des signes. Intermédialités / Intermediality, (27).

https://doi.org/10.7202/1039816ar
Résumé de l'article

Le présent texte s'interroge sur l'espace cognitif à ouvrir afin de rendre possible, au sein de la théorie littéraire et artistique, une réception entière des oeuvres de création en langues des signes. Il propose le développement d'une théorie de l'oeuvre et de la réception comme geste politique nous rendant apte à répondre à l'invitation de Rada Iveković pour parvenir à traduire contre la violence. 


\title{
Le tiers synesthète : espace d'accueil pour la création en langue des signes ${ }^{1}$
}

\author{
Julie Chateauvert
}

1 s'agit par ce texte d'aménager un espace. D'étendre une aire qui autorise les manœuvres nécessaires aux recadrages. Il s'agit de créer l'intervalle qui permette d'introduire sous le regard de la critique littéraire (ou d'art), qui ne la voit qu'exceptionnellement, la création faite dans ces langues ni vocales ni écrites, les langues des signes (LS). Par l'entremise du développement d'outils conceptuels nécessaires à la réception des œuvres, il s'agit, grâce à eux, de revenir sur les instruments d'analyse dont nous disposons déjà afin d'interroger les mécanismes de leur opérationnalité politique. En tête, le désir de favoriser les circulations. En d'autres termes, ce texte constitue la mise en chantier d'une idée de recherche qui, pour parler comme Rancière, interroge un certain partage du sensible ${ }^{2}$.

Rada Iveković m'inspire lorsqu'elle écrit :

Traduire implique au-delà du passage ou du transfert d'une langue en une autre ou d'un lieu en un autre, la vie aux frontières et l'accueil ou l'asile de l'œuvre, des humains, avec une partie de leurs contextes, dans un nouvel environnement. Cet asile crée à son tour un ou plusieurs nouveaux entourages qui se traversent et coexistent 3 .

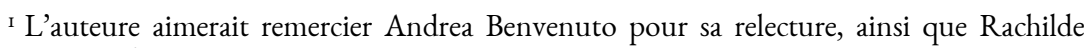
Benelhocine, Émilie Barbier, Claire Danet, Jules Turlet, Ruth Kitchen, Soline Vennetier et Roberta Estimado pour les échanges auxquels ils ont participé lors du séminaire « Travaux en cours des jeunes chercheurs », le 3 mai 2016 à l'EHESS Paris, et qui ont nourri la rédaction de ce texte.

${ }^{2}$ Jacques Rancière, Le partage du sensible, esthétique et politique, Paris, La fabrique éditions, 2000.

3 Rada Iveković, « Que veut dire traduire ? Les enjeux sociaux et culturels de la traduction », Asylon(s), $\mathrm{n}^{\mathrm{o}}$ 7, juin 2009. En ligne, http://www.reseau-terra.eu/rubriquer7r.html (consultation le I9 décembre 2016). 
S'intéresser à la création dans les LS implique d'emblée une traduction multidirectionnelle et continue: une traduction culturelle entre les signants et les parlants ou, en se positionnant autrement, entre la sourditude ${ }^{4}$ et la majorité; une traduction linguistique dont il faudra discuter, en plus de la pratiquer. Cela nécessite également une traduction entre les disciplines, mais plus encore, puisqu'il s'agit d'en éroder les lignes de partage, d'être indisciplinée, en quelque sorte.

La réflexion théorique (encore rare) qui s'est attardée à la création en LS s'est, par exemple, essentiellement construite à partir de la linguistique. Je dis essentiellement non pas parce qu'elle en est uniquement issue, mais parce qu'étant donné l'histoire de la recherche sur les LS et la culture sourde, la linguistique constitue une discipline phare. Ses assises bien ancrées lui confèrent une autorité certaine. Étant donné cette filiation, les études portant sur la création en LS ont eu davantage pour visée, comme pour effet, de préciser les descriptions des langues et, simultanément et en parallèle, de nourrir les Deaf Studies que d'interpeller plus largement la théorie littéraire ou la critique d'art.

La proposition d'Iveković tombe juste. L'objet du présent texte sera donc de s'en inspirer et de fabriquer des espaces d'accueil pour ces œuvres qui stimuleront, je l'espère, la formation de nouveaux entourages. Ainsi, afin de cultiver les conditions propices à leur formation, je propose une réflexion qui se déploie le long de certaines charnières articulant un rapport entre art et politique telles qu'elles se forment dans le contexte particulier des LS.

Nous parcourrons ensemble trois territoires cognitifs. Nous nous attarderons d'abord assez longuement dans le premier, où je présenterai certains éléments du contexte sociopolitique dans lequel survient la création en LS. La conscience de ce contexte sociopolitique, légal aussi, m’apparaît cruciale pour percevoir la portée de l'élaboration d'un discours sur l'art issu des populations locutrices des LS. Puis, traversant dans un second territoire, je proposerai quelques éléments d'épistémologie de la recherche sur la création en LS. Mon objectif sera alors de mettre en lumière la continuité de ce contexte sociopolitique à l'intérieur de cette recherche qui le travaille

${ }^{4}$ La sourditude désigne le processus de constitution d'une identité culturelle sourde qui s'émancipe des structures porteuses d'oppression par la guérison et l'ouverture. Le terme « sourditude » est la traduction la plus souvent utilisée pour désigner le concept de deafhood, au cœur des recherches de Paddy Ladd. Fabrice Bertin, dans l'introduction de Les sourds (20IO), dit que le terme aurait été forgé en s'inspirant du concept de négritude d'Aimé Césaire. Paddy Ladd, Understanding Deaf Culture: In Search of Deafhood, Clevedon, Multilingual Matters, 2003; Fabrice Bertin, Les sourds, une minorité invisible, Paris, Autrement, coll. « Mutations », 2010 . 
en même temps qu'elle est travaillée par lui. C'est à partir de là que je me saisirai de la question de l'intermédialité, m'interrogeant sur le sens esthétique et politique qu'elle revêt lorsqu'elle se manifeste dans les œuvres de création en LS. Finalement, après avoir proposé quelques matériaux pour une appréhension des œuvres, je conclurai en cherchant des brèches, des ouvertures qui permettent d'envisager la pratique de la traduction et de la réflexion théorique à propos de la création en LS comme aménagement de lieux d'accueil, comme pratique de transformation.

\section{Premier TERRITOIRE, CELUI DU POLITIQUe}

\section{Silhouette Des lignes de PARTAGE}

Le contexte importe. Si Iveković invite à l'ouverture de lieux d'asile, c'est bien sur la base d'une conscience d'asymétries préalables, voire de rapports de domination. Elle nous dit :

L'histoire coloniale puis postcoloniale a fait éclater au grand jour les tensions politiques du traduire, de même qu'elle a fait apparaître et exposé les différents points de vue, les contextes et les perspectives décentrées, contradictoires, pluriels et hors champ. Mais ceci vient par-dessus le marché, en quelque sorte, puisque les langues et la traduction portent en elles la pluralité irréductible de l'humains.

Lorsque les LS se forment en objet d'étude, elles occupent un lieu singulier et potentiellement névralgique pour la stabilité de l'édifice d'une pensée qui embrasse beaucoup plus largement qu'elles. Les locuteurs et locutrices des LS se réferent à une appartenance culturelle. Renforcées par une histoire de lutte pour leur reconnaissance, les langues constituent le cour d'une identité à défendre et à affirmer ${ }^{6}$. Le plus souvent liée à l'expérience de la surdité, mais non exclusivement, cette appartenance culturelle

\footnotetext{
5 Rada Iveković, 2009.

${ }^{6}$ La littérature scientifique qui décrit le mouvement pour l'autodétermination d'une identité collective sourde ancrée dans appartenance à une minorité culturelle et linguistique est abondante. Pour s'initier à son histoire, on pourra consulter : Carol Padden et Tom Humphries, Inside Deaf Culture, Cambridge, Harvard University Press, 2006. Padden et Humphries y développent une perspective conçue à partir d'une appartenance à la communauté sourde. On lira également Andrea Benvenuto, Les sourds existent-ils?, Paris, L'Harmattan, 2006. Benvenuto y collige et présente des textes marquants du sociologue Bernard Mottez rédigés entre 1976 et 1996. La quatrième partie de l'ouvrage (p. 309-370), notamment, présente un intérêt particulier pour le présent article, puisqu'elle explore l'idée d'une nation sourde. Si l'on souhaite se limiter plus spécifiquement au Québec ou au Canada, on trouvera une initiation éclairante dans Nathalie Lachance, Territoire, transmission et culture sourde: perspectives historiques
} 
revendiquée vient exercer une pression sur les balises d'une pensée de la transmission par filiation ou par ethnicité7. Par ailleurs, la volonté que démontre la collectivité culturelle sourde de faire défection pour s'affranchir du système référentiel du handicap met également à mal l'appareil normatif qui régit les corps ${ }^{8}$. Dans un monde, cependant, où l'imaginaire des langues ne se décline pratiquement que sur un mode vocal (en admettant sa transposition graphique), tout l'écosystème qui se forme autour des LS comme noyau est aux prises avec un risque constant et transversal d'occultation.

Dans ce contexte, les gens qui occupent le versant dominant du rapport tendu entre la majorité, réputée « normale », et la minorité sourde, n'imaginent pas être ou avoir été acteurs d'une histoire pouvant s'apparenter à une histoire coloniale. La condition physiologique de surdité, liée à l'usage des LS, fait écran. Une portion d'entre eux se voit surtout comme dispensateurs de soins ou de services de réadaptation qui permettent de compenser un déficit. Leur action propose une normalisation célébrée largement dans la population, notamment parce qu'elle fait miroiter la promesse d'une inclusion. Sur l'autre versant, pourtant, celui habité par la minorité culturelle sourde, on a bel et bien recours au champ lexical qui nomme une colonisation et documente ses répercussions tant sur les structures que sur les représentations. Ladd est, pour ces thèses, un auteur de référence9. Il identifie une volonté d'assimilation conquérante lorsqu'il fait le récit du lobby qui mènera à l'interdiction des LS et à leur expulsion hors

7 Sarah Batterbury, Paddy Ladd et Mike Gulliver, « Sign Language Peoples as Indigenous Minorities: Implications for Research and Policy », Environment and Planning A, $\mathrm{n}^{\circ} \mathrm{I2}, 2007$, p. 2899-2915. Les auteurs conçoivent un peuple transfrontalier constitué à partir de la transmission des LS, qu'elle passe ou non par une filiation. La persistance de la présence des LS sur des territoires donnés qui, bien que contraints par les systèmes d'éducation, ne respectent pas obligatoirement les découpages géolinguistiques liés aux territoires nationaux, les amènent à les considérer au même titre que les langues autochtones. En revanche, Lane, Pillard et Hedberg soutiennent la thèse qu'une transmission filiale des LS et de la culture sourde existe néanmoins et qu'une ethnicité sourde peut être conçue en remontant une généalogie sourde organisée en clans. Harlan Lane, Richard Pillard et Ulf Hedberg, The People of the Eye: Deaf Ethnicity and Ancestry, New York, Oxford University Press, 2oII.

${ }^{8}$ Ibid., p. 36-I40. La première partie de l'ouvrage de Benvenuto sera ici utile. Y sont rassemblés les textes de Bernard Mottez discutant de l'« être sourd » et dans lesquels est envisagée la constitution d'une appartenance culturelle sourde face aux pressions normatives innervant la société.

9 Ladd, 2003. Pour une présentation plus synthétique de la façon dont un courant des Deaf Studies s'est constitué en se référant aux études postcoloniales, on pourra lire ce chapitre : Paddy Ladd, « Colonialism and Resistance: A Brief History of Deafhood », dans Humphrey-Dirksen L. Bauman (dir.), Open Your Eyes: Deaf Studies Talking, Minneapolis, University of Minnesota Press, 2008, p. 42-59. 
des établissements d'enseignement. Il en démontre le caractère déterminé lorsqu'on décrit le recours aux coercitions physiques pour empêcher l'expression linguistique. Il souligne également les stratégies de normalisation plus indirectes lorsqu'il relate les manœuvres d'empêchement de regroupement entre locuteurs des LS par le biais de l'intégration en milieu scolaire ordinaire. Il a recours encore au champ référentiel de la colonisation lorsqu'on met en évidence le déni des autorités à octroyer des droits linguistiques, même lorsqu'elles acceptent les LS au registre des langues véritables, voire qu'elles leur concèdent un statut officiel ${ }^{1 \mathrm{O}}$.

La population locutrice des LS s'apparente à une diaspora ${ }^{\mathrm{II}}$ : une diaspora singulière, s'il en est, puisqu'elle est sans territoire originel, mais néanmoins liée transnationalement et soudée par l'imaginaire d'un commun; une diaspora formée d'un peuple qui n'a de cesse d'affronter les tentatives de dissoudre ses lieux de rassemblement constitutif, physiques comme imaginaires. Car c'est hors de l'intelligence, hors même des conditions de réalisation de l'humanité, qu'on a eu l'idée de les expulser ${ }^{12}$.

Si cette dispute traverse l'histoire des idées, elle marque de même une histoire politique, aux effets très concrets, déterminante pour comprendre où intervient la critique esthétique des œuvres. Prenons appui sur le récit d'une trame indispensable de cette histoire politique : en 2010 , lors du XXI International Congress of the Education of the Deaf, le comité organisateur a consacré son allocution d'ouverture ${ }^{\mathrm{I}}$ à

Io Il est impossible ici, sans trop dévier de mon propos, de présenter une synthèse de l'état des droits linguistiques conférés aux LS à travers le monde. Pour se sensibiliser aux enjeux, on se référera au numéro spécial de Sign Language Studies consacré aux politiques publiques en matière linguistique pour les LS. Joseph Quer et Ronice De Quadros (dir.), Sign Language Studies. Special Issue: Language Planning and Policies for Sign Languages, vol. I2. $\mathrm{n}^{\mathrm{O}} 4$, été $2 \mathrm{OI2}$, p. $465-624$.

II Steven D. Emery, «A Deaf Diaspora? Imagining Deaf Worlds Across and Beyond Nations », It's a Small World: International Deaf Spaces and Encounters, Michele Friedner et Annelies Kusters (dir.), Washington, Gallaudet University Press, 2015, p. I87-198.

${ }^{12}$ Kant, pour ne donner qu'un exemple parmi plusieurs, formule cette sentence: « Les sourds de naissance, condamnés par là-même à rester également muets, ne peuvent jamais accéder à plus qu'à un analogon de la raison ». Emmanuel Kant, Anthropologie du point de vue pragmatique [1798], tr. de l'allemand par P. Jalabert, dans CFures philosophiques, t. 3, Paris, Gallimard, Pléiade, 1986, \ I8, p. 974, tel que cité dans Raphaël Ehrsam, « Représentation des sourds et muets et fonctions de la parole de Descartes à Kant », Archives de Philosophie, t. 75, $\mathrm{n}^{\circ} 4,9$ novembre 20I2, p. 643-667.

${ }^{13}$ On peut lire ici la réaction de la Fédération mondiale des sourds à la suite de cette déclaration: «International Congress of the Deaf (ICED), July I8-22, 20Io, Vancouver, 
l'expression de regrets pour les torts occasionnés à la suite des recommandations faites lors de son $\mathrm{II}^{\mathrm{e}}$ Congrès international tenu en $\mathrm{I} 88 \mathrm{o}$ à Milan ${ }^{\mathrm{I}}$. Le président de l'événement, l'abbé Tarra, s'exprimait alors en ces termes :

Speech is addressed to the intellect, while gestures speak coarsely to the senses. I used signs for many years in my religious teaching, but decided definitely to give them up and adopt the pure oral system, because I became convinced that my pupils, instead of understanding the abstract ideas I intended to convey to them, were only placed in possession of grossly material images ${ }^{15}$.

Les conclusions du rapport rédigé au terme de ce congrès soutenaient que seule une méthode d'éducation qui contraindrait les enfants sourds à oraliser une langue vocale saurait garantir leur complète humanité qui, elle, passe par une subsomption des sens en un esprit pur. Ces recommandations viennent légitimer un mouvement déjà en cours au sein des instances législatives de la plupart des nations occidentales et donnent le levier nécessaire aux directions d'établissement adeptes d'une méthode d'enseignement strictement oraliste. En conséquence, l'accès à une éducation en LS reculera notablement, et les retombées de ce congrès seront durables. Au sein des populations locutrices des LS, le congrès de Milan, traumatique, agit comme un mythe fondateur.

Tout au long du siècle qui suit ces interdictions, on assiste, en réaction, à l'organisation de luttes pour la reconnaissance des LS visant à contrer les effets délétères de ces mesures. Ces luttes façonnent la vie de la communauté, dont les démarches artistiques. Progressivement, elles prendront aussi forme à l'intérieur de la recherche universitaire. Aujourd'hui, l'équilibre des forces s'est suffisamment modifié pour que puisse être formulée une demande de pardon. À un échelon institutionnel plus élevé, l'état actuel du rapport de force rend possible l'inscription, dans les chartes et résolutions rédigées au sein des instances de l'ONU, d'une incitation à la reconnaissance des LS comme langue naturelle pour les sourds et le respect de leur droit à recevoir un

Canada », World Federation of the Deaf, 20Io, https://wfdeaf.org/news/internationalcongress-of-the-deaf-iced-july-I8-22-2010-vancouver-canada (consultation le is décembre 2016).

${ }^{14}$ Pour le contexte historique et une synthèse des tenants et aboutissants du congrès de Milan, on pourra consulter Florence Encrevé, «Réflexions sur le congrès de Milan et ses conséquences sur la langue des signes française à la fin du XIX ${ }^{\mathrm{e}}$ siècle », Le Mouvement social, $\mathrm{n}^{\mathrm{o}}$ 2, 2008, p. 83-98.

is Edward Miner Gallaudet, « The Milan Convention », American Annals of the Deaf and Dumb, vol. 26, $\mathrm{n}^{\mathrm{o}} \mathrm{I}, \quad$ janvier I88I, http://www.foundinblank.com/EMG-Milan.html (consultation le is décembre 2016). 
enseignement dans cette langue ${ }^{16}$. Pour sa part et bien qu'elle y voit un levier important pour la conquête des droits en cours, Sarah Batterbury remarque que cette inscription est porteuse d'une double posture. Certes, on y recommande le recours aux LS dans l'enseignement, mais toujours en les maintenant à l'intérieur du cadre normatif du handicap plutôt qu'en les ajoutant aux articles concernant les droits linguistiques ${ }^{17}$. Les positions adoptées au Parlement européen, en 1988 et en 1998, puis par le Conseil de l'Europe en 20oI, sont un peu différentes. On inscrit alors dans la Charte européenne des langues régionales et minoritaires des recommandations à l'intention des États afin que les LS bénéficient d'une protection similaire à celle accordée aux langues régionales et minoritaires d'Europe ${ }^{18}$. Batterbury, Ladd et Gulliver vont plus loin et démontrent la légitimité, pour les LS, d'une reconnaissance des mêmes droits que ceux dont jouissent les langues autochtones ancestrales ${ }^{19}$. Or, bien que des décrets et des lois suivant ces avis aient été entérinés dans quelques États, l'instauration de mesures d'application n'en est pas garantie pour autant. C'est encore souvent hors la loi qu'on enseigne, en LS ou non, le patrimoine de création qui en est issu (si on l'enseigne).

Au Canada, bien que la convention de l'ONU sur les droits des personnes handicapées ait été signée (2007) puis ratifiée (2010), une résistance à la reconnaissance officielle des LS semble résider dans la structure de la constitution du pays elle-même. C'est un «nœud gordien », concluent Émilie Moniz et Mark Power ${ }^{20}$. En effet, au Canada, les LS ne jouissent d'aucun statut juridique ${ }^{21}$. Seules les langues officielles,

${ }^{16}$ Pour une mise en contexte, voir: Annika Pabsch, «UN Convention on the Rights of Persons with Disabilities », dans Genie Gertz et Patrick Boudreault (dir.), The SAGE Deaf Studies Encyclopedia, Thousand Oaks, SAGE Publications, 2016, p. 986-90. Pour le texte de la convention lui-même, voir : Convention relative aux droits des personnes handicapées et Protocole facultatif, http://www.un.org/french/disabilities/default.asp?id=I4I3 (consultation le 30 avril 2014).

${ }^{17}$ Sarah Batterbury, « Language Justice for Sign Language Peoples: The UN Convention on the Rights of Persons with Disabilities », Language Policy, vol. II, $\mathrm{n}^{\circ}$ 3, août 20I2, p. 253-72.

I8 « Droits des minorités nationales - Recommandation 1492 (200I) de l'Assemblée parlementaire REC_I492 (200I) et GR-H(2002)CB8 », Conseil de l'Europe, I3 juin 2002, https://rm.coe.int/CoERMPublicCommonSearchServices/DisplayDCTMContent?document $\mathrm{Id}=09000016804 \mathrm{e} 698 \mathrm{f}$ (consultation le 20 décembre 20I6).

19 Batterbury, Ladd et Gulliver, 2007, p. 2899-2915.

${ }^{20}$ Émilie Moniz et Mark Power, « Langues des signes au Canada : entre droits linguistiques et droits de la personne », Mc Gill Journal of Law E\& Health, vol. 8, no I, 20I4, p. 43-78.

${ }^{21}$ Notons qu'il existe, au Canada, cinq LS différentes : l'American Sign Language (ASL), la Langue des signes québécoise (LSQ), la Plain Indian Sign Language (PISL), la Langue des signes des Maritimes (MSL) et la Langue des signes inuite (IUR). Seules les deux premières sont 
l'anglais et le français, bénéficient de droits linguistiques comprenant notamment leur protection lorsqu'elles sont parlées sur des territoires où elles sont minoritaires ${ }^{22}$. La jurisprudence comprend bien quelques jugements par lesquels on a octroyé des droits linguistiques partiels aux locuteurs des LS. Ceux-ci ont cependant été obtenus, dans certains cas particuliers, en invoquant l'article is de la Charte canadienne des droits et libertés. Or, cet article a pour fonction de protéger les citoyens contre la discrimination en raison d'un handicap physique. En effet, afin de favoriser l'égalité réelle entre les individus, on interprète alors l'article de manière à autoriser le recours à des interprètes en LS. Cependant, comme l'énoncent Moniz et Power $^{23}$ : «En reconnaissant les LS uniquement comme moyen d'enrayer la discrimination et de favoriser l'égalité des personnes sourdes et malentendantes, la réalité culturelle de ces langues et de ces locuteurs n'est pas prise en compte ${ }^{24} \gg$.

En effet, étant donné la formulation des articles concernés de la Charte ${ }^{25}$, pour se voir reconnaître les mêmes droits linguistiques que ceux des langues officielles en situation minoritaire, il faudrait accepter de considérer les LS comme des extensions des langues vocales, et ce, en dépit de leur volonté d'affirmation, ce qui revient à instituer leur subordination. Voie sans issue. Au Canada, la loi constitutionnelle ellemême contraint ainsi les locuteurs des LS à choisir l'aliénation pour éviter la discrimination.

On imagine la stupéfaction des constituants s'ils prenaient conscience d'avoir exclu de leur réflexion l'idée qu'une langue n'est pas obligatoirement de modalité vocale, faisant ainsi l'impasse sur cette pluralité irréductible de l'humain qu'évoque Iveković. C'est cette même stupéfaction qui saisit toutes celles et ceux demandant invariablement, au moment de la découverte des LS, s'il n'y a pas là un code universel : expression paradoxale qui, à la fois, projette le désir humaniste ultime de communication intégrale et signifie, du même souffle, son incapacité à considérer les

considérées vigoureuses, les autres étant signalées comme en danger d'extinction. Les données statistiques les concernant peuvent être consultées sur le site Ethnologue. Languages of the World, www.ethnologue.org (consultation le 20 décembre 2016).

${ }^{22}$ Pour leur part, les langues autochtones se voient octroyer certains droits linguistiques limités. Dans les Territoires du Nord-Ouest ainsi qu'au Nunavut, par exemple, les langues autochtones se sont vues conférer un statut de langue officielle. Ce statut est lié à un territoire, et les langues autochtones parlées ailleurs au Canada ne bénéficient pas de cette reconnaissance. Pour le texte de la Charte des droits et libertés du Canada, consulter: http://lawslois.justice.gc.ca/fra/const/page-Is.html.

${ }^{23}$ Moniz et Power, 2014, p. 47.

24 Ibid.

${ }^{25}$ Il s'agit des articles I6 à 20 de la Charte canadienne des droits et libertés. 
LS au même titre que les langues vocales. Dans les deux cas, il s'agit bien d'une éclipse cognitive qui fonde, en représentations comme en droit, une clandestinité.

\section{DEUXIÈme TERRITOIRE, CELUI DE L’éPISTÉMOLOGIE}

\section{ÉPISTÉMOLOGIE EMBARRASSÉE : POURQUOI (COMMENT ?) S'INTÉRESSER À LA CRÉATION EN LS ?}

J'écris ce texte, en somme, dans le contexte d'une stupéfaction générale; dans un temps où la curiosité fréquemment manifestée à l'égard des LS force, du même coup, le constat de l'inégalité qui prévaut entre les langues. Témoin de l'asymétrie $\mathrm{du}$ rapport, ce constat fait par Clayton Valli 26 : dans l'intervalle entre les interdictions et le moment où les luttes pour la reconnaissance parviennent à impulser un mouvement de transformation dans les représentations, les poètes de langue première signée ont souvent d'abord créé en leur langue de scolarisation. Peu à peu, ensuite, et comme l'affirmation culturelle et identitaire gagne en force, les artistes commencent à créer directement en LS. Nourrissant en boucle le vœu d'émancipation, ces créations renforcent le sentiment d'appartenance et galvanisent le mouvement d'affirmation. En d'autres termes, et pour évoquer de nouveau Rancière, ces artistes engagent les formes pour déplacer les lignes de partage du sensible et ainsi métamorphoser le commun.

En parallèle, au sein de la majorité, des gens ont aussi voulu agir, se rapprocher. Ainsi, nous nous sommes mis à traduire. La manœuvre est délicate, et on peut se demander si les démarches de traduction n'ont pas maintes fois tendu vers l'assimilation ${ }^{27}$.

${ }^{26}$ Clayton Valli, « Poetics of American Sign Language Poetry », The Union Institute Graduate School,1993, p. 25-39. En plus de l'historique proposé par Clayton Valli, on visionnera, pour des témoignages d'artistes sourds racontant leur parcours de création : Myriam Lerner et Don Feigel, The Heart of the Hydrogen Jukebox, DVD, Rochester, Rochester Institute of Technology, 2009. On y voit notamment Patrick Greybill et Peter Cook, deux artistes en LS jouissant d'une renommée internationale, relater le moment de leur prise de conscience de la possibilité de créer dans leur propre langue.

${ }_{27}$ Pour le lectorat familier avec le contexte sociopolitique dont il est question, il est utile ici de préciser le corpus auquel je fais référence. Je m'intéresse particulièrement au corpus qu'on pourrait qualifier de littéraire en faisant la transposition des catégories de genre auxquelles on a recours pour les langues vocales et écrites : la poésie et le récit plus spécifiquement. J'exclus ici la tradition d'œuvres théâtrales et la production de vidéoclips reprenant des chansons populaires et les adaptant en LS. 
En gros et vu du côté positif, [la traduction,] c'est le pouvoir de désamorcer la violence en faisant passer des idées d'un contexte à un autre. N'oublions pas que faire passer les idées, c'est aussi faire passer des humains - ceux qui les créent, qui les portent, qui les traduisent, qui les font passer en contrebande, qui les imposent, qui les subissent, qui les adoptent ou s'y fourvoient... Il va de soi que la traduction porte en soi le même potentiel de violence que de non-violence ${ }^{28}$.

Inévitablement empreint du contexte sociopolitique, chaque projet de traduction se trouve d'emblée adossé à ces lignes de partage du sensible qui ordonnent la cohabitation des signants et des parlants. L'érodera-t-il ou le confirmera-t-il ? Parvenons-nous à traduire contre la violence ${ }^{29}$ ?

Depuis les démonstrations faites par William Stokoe rétablissant un statut de langues naturelles pour les $\mathrm{LS}^{30}$, on a beaucoup traduit des langues écrites vers elles, entre autres poussé par le désir de rendre accessible, pour la minorité considérée en déficit de culture, la littérature de la majorité. La prise de conscience par cette dernière qu'il existe aussi chez la minorité une création originale, patrimoniale comme actuelle, semble demander temps et disponibilité d'esprit. Possiblement le temps de se remettre de la stupéfaction, notamment, puis celui nécessaire pour se doter d'outils analytiques adéquats $^{3 \mathrm{I}}$. Alors qu'on remarque que les traductions vers une langue écrite d'œuvres

\footnotetext{
${ }^{28}$ Rada Iveković, 2009.

29 Lors d'une journée d'étude portant sur la traduction entre la LSF et le français organisée à l'Université Paris 8 en novembre 20I5, une participante, elle-même sourde et locutrice de la LSF, est intervenue pour interpeller l'assemblée et lui exprimer son irritation face à l'empressement de la majorité entendante à s'adonner à des projets de traduction, ramenant ainsi l'attention constamment vers les langues vocales ou écrites. Elle soutenait qu'il était primordial de soutenir d'abord et avant tout la création originale en LS. J'ai été plusieurs fois témoin d'interpellations allant en ce sens lors de différents rassemblements ou ateliers mixtes ou organisés par les communautés sourdes auxquels j'ai eu la chance d'assister. Ces interventions nous informent, à mon avis, du déséquilibre des forces en présence et méritent qu'on réfléchisse avec sérieux et attention à cette question.

30 William Stokoe est réputé pour être le linguiste qui a amorcé le mouvement de retournement contrant les effets du congrès de Milan en publiant une première étude confirmant, au sein de sa discipline, le statut de langue véritable pour l'American Sign Language. William C. Stokoe, Sign Language Structure: An Outline of the Visual Communication Systems of the American Deaf, Silver Spring, Linstok, 1960.

${ }^{31}$ Dans ma thèse de doctorat, j'ai proposé une recension des textes théoriques forgeant l'émergence d'une critique pour la création en LS qui suit également le fil de la transformation des représentations. Julie Chateauvert, Poétique du mouvement: ce que les LS font à la littérature, Montréal, Université du Québec à Montréal, 2014, ainsi qu'en ligne, www.poetiquels.net.
} 
poétiques créées en une LS sont relativement peu fréquentes, on constate aussi que leur réalisation nécessite d'apprendre à voir les œuvres dans toute leur complexité, dans toute leur subtilité 32 .

Or, si la stupéfaction peut se déliter dans l'esprit de qui pratique la traduction ou la conceptualisation de théories, elle ne se dissout pas instantanément, et la transformation des idées ne se fait pas sans être lestée par le contexte. Ainsi, la théorie portant sur la création en LS a ses jurisprudences, pour ainsi dire. Comme les avancées se construisent sur les héritages, elles peuvent également nécessiter de dissoudre des adhérences. Dans le cas qui nous occupe, l'histoire de la théorie est aussi traversée par l'histoire de la quête d'émancipation des populations locutrices des LS. Il s'y joue des efforts de décolonisation :

Sign linguistics, in what we might term the "first wave" of Deaf Studies, concentrated on identifying how signed languages displayed linguistic equivalents of spoken language features, an inevitable first step toward validation and thus decolonization. However, if we are to progress further, a formal move toward identifying the differences between signed and spoken languages (especially the positive powers within signed languages) is necessary if we are to delineate the full dimensions of the Deafhood experience. Nineteenth-century Deaf writers wrote of sign languages as an art rather than a science, making reference to the plasticity of the language, its unique mutability 33 .

Du côté de la théorie, comme du côté de la création ou de la traduction, certains répondent à cet appel. Le modèle de description linguistique de Christian $\mathrm{Cuxac}^{34}$, par

32 J'aimerais attirer l'attention ici sur le travail de l'association Arts Résonances qui, depuis 2008, anime une scène en langue des signes française à l'occasion du festival de poésie Voix Vives dans la ville de Sète. Fruit de la collaboration entre interprètes en LS, poètes signeurs et francophones, des œuvres sont alors traduites dans les deux directions. L'association anime aussi un laboratoire de recherche sur la traduction poétique qui rassemble des poètes, des chercheurs universitaires, des interprètes et d'autres passionnés pour des sessions de travail périodiques auxquelles j'ai eu la chance de participer. Brigitte Baumié, directrice de l'association, a assemblé une anthologie d'une cinquantaine de textes, dont trois sont traduits de la LSF vers le français. Brigitte Baumié, Les mains fertiles: so poètes en langue des signes, Paris, Éditions Bruno Doucey, 2015.

33 Ladd, 2008, p. 5 I.

34 Pour une présentation des différents modèles de description de la LS française, on se référera à Marion Blondel et Leila Boutora, « Description linguistique de la langue des signes française », dans Annelies Braffort (dir.), La langue des signes française (LSF). Modélisations, ressources et applications, Londres, ISTE Éditions, 2016, p. 19-46. Ce chapitre permet, entre autres, de situer le modèle de Cuxac, auquel je réfere ici, parmi d'autres courants. Bien que le chapitre concerne en particulier la LSF, il sert mon propos dans la mesure où il permet de comprendre les articulations entre les différents modèles descriptifs des LS en général et de saisir les enjeux sociohistoriques qui les déterminent. 
exemple, fait des capacités iconiques des langues le pivot du système sémiotique caractéristique des LS ${ }^{35}$. Jolanta Lapiak, pour sa part, développe dans ses œuvres, comme dans ses écrits théoriques, une perspective appelant à la déconstruction d'un phonocentrisme, dominant dans la société, qui marque la réception des œuvres comme la recherche scientifique et qui enserre les processus de création ${ }^{36}$. En 20I4, elle a facilité la formulation d'un manifeste appelant les artistes sourds à libérer leur création de ces marqueurs idéologiques intériorisés ${ }^{37}$.

Selon certains théoriciens et théoriciennes, cependant, ce qui a posé des jalons pour une libération pendant un temps peut freiner le progrès une fois venue l'étape subséquente. Kyra Politt, par exemple, formule une critique de l'héritage de la linguistique structurale qui, lorsque convoquée pour analyser des œuvres de création, entraîne, selon elle, une distorsion du regard. Elle affirme qu'à vouloir reconnaître dans les œuvres créées en LS ce qu'on connaît déjà, qu'à chercher à les inviter entre des balises moulées, quitte à équarrir les bords pour réussir l'enchâssement, on risque de décortiquer les œuvres sans les voir. Ainsi en va-t-il de leur classification sous le genre de la poésie comme il est courant de le faire ${ }^{38}$.

${ }_{35} \mathrm{Au}$ moment de publier la première étude qui allait contrer la disqualification des LS et les faire accepter comme langues véritables par la communauté scientifique, W. Stokoe a dû démontrer qu'elles satisfaisaient à la règle de la double articulation. Il fallait pour ce faire minimiser les caractéristiques iconiques des langues considérées, à l'époque, comme critère d'exclusion des systèmes linguistiques. L'institutionnalisation des LS a dû passer par la démonstration de leur similarité et de leur conformité avec les règles établies de constitution des langues vocales. Il a fallu attendre l'évolution des théories pour être en mesure de réhabiliter l'iconicité comme élément structurant pour les LS et pouvoir avancer des analyses différentialistes sans risquer leur disqualification. Le cadre théorique développé par Christian Cuxac participe à cette réhabilitation de l'iconicité.

${ }^{6}$ Pour découvrir la démarche artistique de Jolanta Lapiak, on visitera www.lapiak.com (consultation le 25 janvier 2017). Ses réflexions théoriques en cours sont, pour leur part, colligés sur le site Handspeak: Sign Language Resource Online, Jolanta Lapiak, www.handspeak.com (consultation le 25 janvier 2017).

37 Formulé au terme d'une résidence pour artistes organisée par SPiLL-PROpagation, on retrouve le manifeste en LSQ, en ASL, en français et en anglais ainsi que le contexte de sa production: «Phonocentrisme: Une déconstruction des pratiques artistique Sourdes au Canada/Phonocentrism: A Deconstruction of Deaf Art Practices in Canada », SPiLLPROpagation, http://spill-propagation.ca/sectionspecialephonocentrism/openhousepage.html (consultation le 25 janvier 2017).

${ }^{38}$ Kyra Politt, « Does Changing the Descriptive Discourse Alter the Art Form? The Issue of Language in Sign Language Poetry », communication présentée lors du colloque 
Si on accepte cette critique, et pour répondre à l'encouragement d'Iveković, comment alors traduire ces œuvres contre la violence ? La question, dès l'amorce, force une suspension : il faudrait d'abord mieux savoir les voir.

\section{TROISIÈME TERRITOIRE, CELUI DE L'AGIR}

LA CRITIQUE D'ART À L'đEUVRE

POUR LA DÉCOLONISATION DES IMAGINAIRES

C'est ici que le développement d'une critique pour les œuvres en LS entre en jeu. Afin que traduire puisse être un geste qui aménage des espaces d'accueil, il nous faut encore créer l'espace cognitif dans lequel ces ouvres pourront déployer toutes leurs facettes. Comment donc agir sur les idées de manière à créer cet espace qui nous permettrait de les recevoir pleinement ? Plus précisément, comment, dans la pratique de la théorie, percevoir avec justesse les formes proposées par ces artistes qui travaillent et assouplissent les lignes de partage et, leur joignant le pas, embrasser la transformation? En ce sens, la fabrication d'une critique pour les œuvres en LS représente à la fois une fête et une responsabilité : la fête d'accueillir une forme d'art au sein de disciplines qui ignorent a priori tout d'elle, et la responsabilité d'esquiver la manière qui reproduit l'asymétrie du pouvoir ${ }^{39}$. Afin, ultimement, de parvenir à faire bouger ces lignes de partage du sensible, les démarches théoriques doivent poursuivre leur travail de description des œuvres en veillant à complexifier le regard. Nous avons également besoin qu'elles s'enrichissent d'une théorie de la réception.

\footnotetext{
Représentations et discours du handicap: l'apport des Disability Studies en sciences humaines, Paris-Sorbonne, 2I novembre 20I5.

39 Un lectorat non initié pourra se familiariser avec la création à laquelle je réfere ici en visionnant, par exemple, le récital donné en ouverture du Festival Phenomena à Montréal en 2012 par le Flying Words Project, Festival Phenomena, Sala Rossa, Montréal, I9 octobre 20I2, http://www.poetiquels.net/Recital\%2odu\%2oFlying\%2oWords\%2oProject (consultation le 20 février 2017) ou encore celui organisé au Swarthmore College par Rachel Sutton-Spence, également en 2012: «Signing Hands Across The Water: An International Festival of Sign Language Poetry from the United Kindom and the United States », Swarthmore College, Swartmore, I7 mars 20I2, http://signinghandsacrossthewater.com/3-I7-I2-performance/ (consultation le 20 février 2017). Une simple recherche sur un moteur de recherche en utilisant les mots clés «sign language » et «poetry», et leurs dérivations en anglais, en français ou d'autres langues permettra de découvrir une multitude de propositions artistiques.
} 
D'ABORD, DU CÔTÉ DE L'đEUVRE :

PRENDRE ACTE DE L'INTERMÉDIALITÉ

Selon Politt, les approches héritières de la linguistique structurale, qui proposent de définir la poésie en LS par le biais de la description de procédés stylistiques, laissent l'essentiel dans l'angle mort. Trop absorbées par le linguistique, trop rompues à des stratégies de segmentations descriptives, ces approches se révèleraient incapables de tenir compte de l'ensemble des composantes esthétiques des œuvres ${ }^{40}$. Politt prolonge, par cette critique, l'appel de Ladd à une décolonisation de la recherche sur les LS. Elle y ajoute que ce que les approches linguistiques ne couvrent pas lorsqu'elles se penchent sur des objets de création, c'est aussi leur fonctionnement comme ouvres d'art. En phase tant avec la critique qu'elle formule qu'avec l'intuition conséquente de la nécessité de se doter de cadrages analytiques à plus large spectre, je propose ailleurs de regarder ces œuvres comme des objets artistiques intrinsèquement intermédiaux ${ }^{4 \mathrm{I}}$.

Une œuvre narrative en LS comprend une dimension linguistique, cela va de soi, puisqu'il s'agit de raconter. Celle-ci est cependant formée également d'autres composantes esthétiques que la description de procédés stylistiques appliqués sur le plan lexical, morphosyntaxique ou prosodique ne suffit pas à saisir. Les LS sont des langues du corps en mouvement, dotées de fortes capacités à faire image et charpentant des récits en structurant un espace de signation ${ }^{42}$, tout autant que ne le font l'espace scénique ou celui de l'écran, selon la destination de diffusion de l'œuvre. Ces composantes ne sont pas uniquement des outils au service de la grammaire, elles sont mobilisées comme autant de médiums artistiques. Si l’artiste en LS reste, de toute évidence, attaché au maniement des structures linguistiques de sa langue, ne relever le geste artistique qu’à ce niveau (ou en premier lieu à ce niveau) nous fera échapper, par exemple, la charge expressive du geste qui nait d'une initiation d'un mouvement au niveau du diaphragme plutôt qu'à celui de

40 Kyra Politt, Signart:(British) Sign Language Poetry as Gesamtkunstwerk, doctoral dissertation, University of Bristol, 20I4, p. I4-I5.

${ }^{4}$ Julie Chateauvert, «Création en langue des signes : intermédialité et proxémie ou La démarche artistique de Jolanta Lapiak : faire comme si nous étions déjà libres », dans Les Actes $d u$ colloque «Représentations et discours $d u$ bandicap, l'apport des Disability Studies en sciences bumaines », Paris, Éditions Garnier, (à paraître).

${ }^{42}$ On appelle « espace de signation » l'espace dans lequel, en situation conversationnelle, se réalise le discours en langue des signes. Il est généralement contenu, sur le plan vertical, entre les hanches et une balise située légèrement au-dessus de la tête. Sur les plans horizontal et sagittal, l'espace de signation se déploie devant le corps à une distance moyenne du tronc, c'est-à-dire, pour une exécution confortable sans extension complète des bras. 
l'épaule; elle nous fera négliger la logique d'un enchaînement qui découlerait du passage d'une composition graphique à une autre, récoltant une suite narrative davantage que si on l'avait réalisée selon un vouloir-dire préalable et intentionnel. Or, nous manquons d'outils analytiques pour parvenir à appréhender la composition des œuvres en des termes qui correspondent aux médiums artistiques. Pour pallier ce manque, je propose de les imaginer composées de quatre pôles attracteurs principaux, précisés de quatre pôles secondaires. Ainsi, les œuvres peuvent être analysées selon leur investissement variable d'un pôle « corps en mouvement », d'un pôle « image » — donnant naissance à deux pôles secondaires, l'un «pictural », l'autre « cinématographique »-, d'un pôle « espace » - donnant naissance à deux pôles secondaires, nommés « espace scénique » et « espace de signation » - et d'un pôle «linguistique ${ }^{43}$. J'adopte l'idée de pôle attracteur pour permettre une description plus flexible et fluide des œuvres que ne le permettent les catégories. En effet, décrire les œuvres en termes d'arrangements interdépendants de composantes esthétiques nous permet de les voir mouvantes et de décrire la relation dynamique que l'artiste établit entre ces composantes tout au long du déroulé de l'œuvre. Il s'agit, en somme, de chercher à développer un système de qualification des œuvres qui reflète leur organisation.

Afin d'illustrer mon propos, bien que partiellement, je suggère d'explorer sommairement une œuvre du Flying Words Project (FWP) 44 créée et interprétée par Peter Cook et Kenny Lerner. L'œuvre est intitulée Wise Old Corn (voir la figure I).

43 Des premiers matériaux pour cette approche ont été présentés lors d'un colloque international en étude du geste. Julie Chateauvert, « The Gravity of Movement: Movement Analysis as a Tool for the Analysis of Creative Sign Language », communication présentée dans le cadre du Symposium Quick on the Draw: Exploring the Multimodal Body through Signart, $7^{\text {th }}$ Conference of the International Society for Gesture Studies, Paris, 2I juillet 2016.

44 Le Flying Words Project (voir http://www.fwpI.com) est un duo de création en ASL créé en 1984 à Rochester. Au fil des ans, le duo s'est acquis une renommée internationale tant comme artistes que comme formateurs. On trouvera utile ici de savoir que les œuvres sont d'abord créées en ASL. Un support de l'anglais, qui n'est pas une traduction, est ensuite ajouté afin de permettre à un public néophyte de suivre le récit tout en restant concentré sur la performance en ASL. Dans le cadre de cet article, cependant, je ne m'attarderai pas à la stratégie de coexistence des langues privilégiée par le FWP. Je référerai uniquement au versant en ASL dans le but de présenter une approche de lecture et d'analyse des œuvres. Je suggère également aux lectrices et lecteurs non locuteurs des LS quelques visionnements sans le support de l'anglais afin de se familiariser avec la matérialité de l'œuvre interprétée en ASL. Les œuvres étudiées ici ont été présentées en ouverture du festival Phenomena, le 19 octobre 2012, à Montréal. Le récital a été organisé en collaboration avec la compagnie Les filles électriques. Son organisation faisait partie du volet intervention de ma thèse de doctorat en études et pratiques des arts (UQAM). 


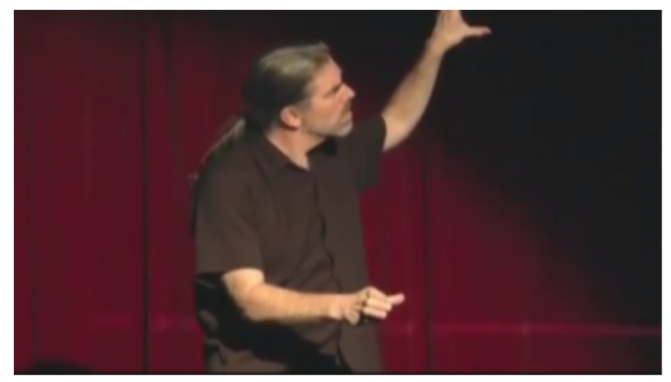

Fig. I. Extrait du récital donné en ouverture du Festival Phenomena à Montréal en 2012 par le Flying Words Project, Wise Old Corn, interprété par Peter Cook et Kenny Lerner, Festival Phenomena, Sala Rosa, Montreal, I9 octobre 2012. Reproduit avec l'aimable autorisation de Flying Words Project, http://erudit.org/media/im/I039816ar/I039816arvooi.mp4.

Globalement, cette œuvre - comme l'ensemble de la démarche de Peter Cook comme auteur et comme performeur - se caractérise par un très fort investissement des pôles «image » et «corps en mouvement», qui tout au long de l'œuvre demeurent dominants. Afin de se familiariser tant avec les capacités iconiques des LS qu'avec l'approche, je propose d'abord de diriger le regard sur les éléments les plus facilement perceptibles par un œil non initié. Ainsi, je me concentrerai sur quelques éléments qui permettent d'envisager par quoi est investi ce pôle «image 45 ». Ces premières observations permettront, dans le même temps, d'identifier quelques formes d'intermédialité fréquemment mobilisées dans la démarche du FWP.

Tout d'abord, on retrouve, dans plusieurs des œuvres du duo, de multiples citations d'images qui ont marqué la culture populaire ou d'œuvres en art visuel. On reconnaitra dans cet extrait, par exemple, l'image fortement médiatisée des athlètes Tommie Smith et John Carlos levant un poing ganté de noir, signe de ralliement des Black Panthers, lors de la réception de leurs médailles aux Jeux olympiques de Mexico en 1968 (voir la figure 2).

45 Chateauvert, 20I4, p. I7I-194. Un texte complet consacré à une analyse d'œuvre serait nécessaire afin d'approfondir la présentation du modèle qui, du reste, est en cours d'élaboration. Compte tenu des limites de cet article, je me contenterai de premiers matériaux en abordant une seule des composantes esthétiques des œuvres en LS. Pour explorer plus avant, les lecteurs peuvent se référer à ma thèse de doctorat, où tout un chapitre est consacré à ce que j'appelle maintenant le pôle « corps en mouvement ». 


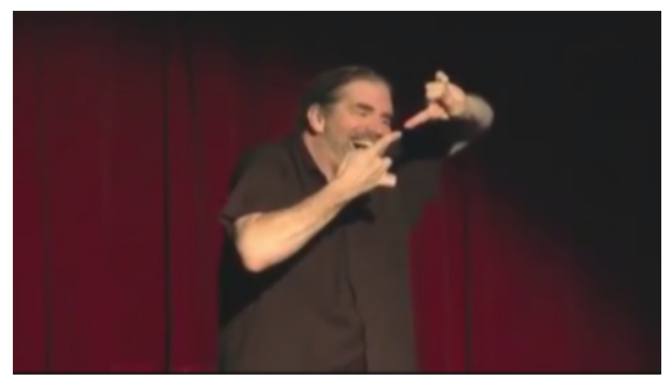

Fig. 2. Extrait du récital donné en ouverture du Festival Phenomena à Montréal en 20 orz par le Flying Words Project, Wise Old Corn, interprété par Peter Cook et Kenny Lerner, Festival Phenomena, Sala Rosa, Montreal, I9 octobre 20I2. Reproduit avec l'aimable autorisation de Flying Words Project, http://erudit.org/media/im/I039816ar/I039816arvoo2.mp4.

Par ailleurs, pour interpréter ce qu'on convoque ici par le rapprochement des deux index - la «Création d'Adam », fragment de la fresque de Michel-Ange peinte au plafond de la chapelle Sixtine - , il est nécessaire de laisser apparaître l'image à l'esprit au-delà du sens littéral de la séquence ${ }^{46}$. La suggestion prend forme, chez le spectateur, incitée par une circularité du récit qui, en boucle, relie itérativement l'ordre du terrestre à celui du cosmique (voir la figure 3 ).

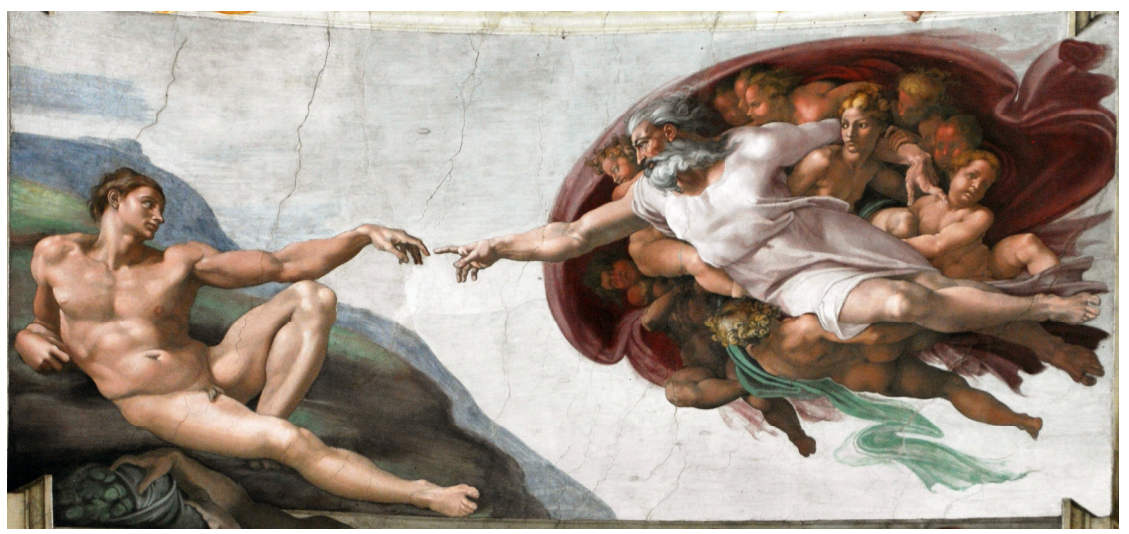

Fig. 3. Michel-Ange, La Création d'Adam, I508-I512, fragment de la fresque peinte au plafond de la chapelle Sixtine, Vatican.

${ }^{46}$ Je laisse ici la lectrice ou le lecteur non initié à l'ASL se rapporter au support offert, en anglais, par Kenny Lerner. Une traduction en français de ce support a été projetée en surtitres lors du récital présenté à Montréal. Elle était formulée comme suit : « Elle germe/sous le soleil/et une fleur/se touche presque ». La traduction est la mienne, faite en collaboration avec Kenny Lerner. 
Un autre degré d'intermédialité traverse, comme une colonne vertébrale, l'œuvre du FWP de part en part. Si, pris largement, elle est littéraire en ce qu'elle travaille la langue, sa composition repose sur des techniques qui dialoguent avec le cinéma : angle de prise de vue, valeurs de plan, effets de caméra subjective, accélérés ou ralentis, parfois même évocations d'effets spéciaux. Une autre séquence de Wise Old Corn permet de percevoir aisément la manœuvre. On y évoque les marches de Selma à Montgomery en 1965 qui, au temps fort de la lutte pour les droits civiques aux ÉtatsUnis, ont été fortement réprimées ${ }^{47}$. Peter Cook nous les donne à voir comme au cinéma. Il le fait si clairement, du reste, que la séquence pourrait, tout autant qu'elle narre l'événement, référer à un extrait d'enregistrement vu par les auteurs et rejoué devant nous: simultanément au théâtre, comme en texte, comme au cinéma. D'ailleurs, par un jeu de mise en abyme, Cook nous le suggère. En effet, il reprend, immédiatement après, une portion significative de la séquence, nous la désignant, cette fois, comme étant captée par un satellite et diffusée sur un écran de télévision (voir la figure 4 ).

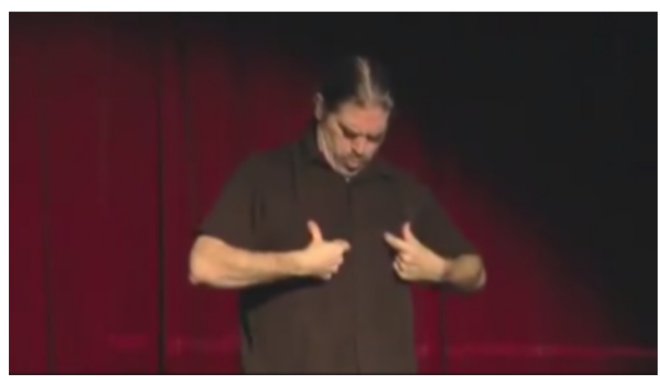

Fig. 4. Extrait du récital donné en ouverture du Festival Phenomena à Montréal en 2012 par le Flying Words Project, Wise Old Corn, interprété par Peter Cook et Kenny Lerner, Festival Phenomena, Sala Rosa, Montreal, 19 octobre 2012. Reproduit avec l'aimable autorisation de Flying Words Project, http://erudit.org/media/im/I039816ar/1039816arvoo3.mp4.

Une part de la critique qu'adresse Politt aux analyses d'œuvres réalisées à partir de l'héritage de la linguistique structurale est de ne pas être en mesure de rendre compte pleinement de telles dimensions. Frank Bechter, pour sa part, formule la même idée dans une critique à plus large portée et qui concerne l'ensemble des recherches consacrées aux LS :

47 Pour plus de contexte sur les marches de Selma à Montgomery, une simple recherche sur internet permettra de trouver de nombreuses vidéos de documentation. On cherchera également plus spécifiquement de la documentation sur la marche du 7 mars 1965, nommée Bloody Sunday, en raison de la violence de la répression policière qu'ont subite les marcheurs. 
Deaf Studies faces a discursive landscape that was not designed for it. The discourses of linguistics, anthropology, literary theory, literacy theory, multiculturalism, etc., are the very platforms for being seen and heard on the public stage; they are the 'language' offered to Deaf Studies for writing itself. But, insofar as these discourses were designed for nondeaf people, and have evolved in harmony with nondeaf values, the story seems rigged from the $\operatorname{start}^{4}{ }^{8}$.

L'œuvre de Peter Cook, en offrant une résistance à ces cadres théoriques préalables, exerce une pression sur les lignes de partage du sensible. Elle met au défi, par le fait même, le travail de traduction potentiel qui s'y appuierait. Si, à défaut d'un cadre de référence capable d'appréhender les œuvres sans les contraindre dans des balises non conçues pour elles, nous ne parvenons pas à les voir dans toute leur complexité, que devient la traduction proposée ? Et dès lors, comment traduire contre la violence ?

ENSUITE, DU CÔTÉ DE LA RÉCEPTION:

LA QUESTION DU PASSAGE

Nous voici à l'approche de latitudes encore moins explorées. Du côté de la théorie existante, on a surtout cherché à décrire les rouages de structuration des œuvres. Nous sommes cependant toujours singulièrement dépourvus de réflexions qui portent sur l'expérience sensible de leur réception ${ }^{49}$. Avec toujours à l'esprit, comme en filigrane, cette invitation à traduire contre la violence, on s'interroge: quelles inspirations nous viendraient d'une attention délicate aux sensations que ces œuvres, toutes intermédialités considérées, font naître chez qui les reçoit ? Comment agissentelles sur nos perceptions, nous affectent-elles? Comment cette intermédialité constituante des œuvres passe-t-elle de la performance de l'artiste à mon expérience comme spectatrice? Comment une théorie de la réception peut-elle enrichir notre aptitude à percevoir les œuvres et, ainsi, agir de concert avec ces artistes qui repoussent les lignes de partage du sensible?

${ }^{48}$ Frank Bechter, «The Deaf Convert Culture and Its Lessons for Deaf Theory », dans Humphrey-Dirksen L. Bauman (dir.), Open Your Eyes: Deaf Studies Talking, Minneapolis London, University of Minnesota Press, 2008, p. 7I.

49 Politt, 2014, p. 55-58. L'auteure crée en ce sens une ouverture intéressante lorsqu'elle convoque la phénoménologie de Merleau-Ponty pour que la compréhension des œuvres en LS s'enrichisse d'une attention portée à l'ensemble de ses composantes esthétiques. Si elle ouvre ainsi à une approche par le biais du sensible qui la distingue des approches plus structurelles, elle demeure cependant du côté de l'œuvre, revisitant la philosophie des sens afin d'interroger la réception sensible du monde par une personne sourde, physiologiquement et culturellement, et la façon dont celle-ci se traduira dans sa création, si elle est artiste. Plus particulièrement, Politt se réfere au concept de Deaf Visualities développé par Ernst Thoutenhoofd. 
J'aimerais penser ici avec Liliane Louvel, avec précaution, sans être trop empressée et en gardant en tête les appels à la vigilance formulés au sein des Deaf Studies qui mettent en garde contre les pièges tendus par l'application aux LS de grilles d'analyse non pensées pour et avec elles. On trouve, néanmoins, dans Le tiers pictural, pour une critique intermédiale de fortes connivences avec ce que l'on tente de saisir iciso $^{\text {so }}$ Il se trouve, dans ce livre, une approche qui fait écho au désir exprimé ici de développer un regard sachant honorer les œuvres en LS. En somme, Louvel suscite mon inspiration au moment fragile où il s'agit de commencer.

Son cadre épistémologique n'est pas celui d'où émerge ma propre réflexion, mais nos regards se croisent. Louvel consacre cet ouvrage à l'étude de la relation entre le texte et l'image. Elle s'intéresse aux évocations de la peinture dans la littérature et, à partir d'elles, propose «d'examiner comment [...] des notions techniques propres au médium (de la peinture) et à son histoire : perspective, cadres, cadrages [...], véronique, nature morte, portrait, figure $[\ldots]$, peuvent faire système et constituer un outil opératoire pour ouvrir [...] "l'œil du texte" ${ }^{51} \gg$. La démarche, déjà, est intéressante, puisqu'il s'agit également pour nous, et en la paraphrasant, de constituer un outil opératoire qui puisse ouvrir non seulement l'œil, en se référant au cinéma par exemple, mais la kinesthésie, l'haptique et la proprioception du texte (si le vocable est toujours adéquat lorsqu'on parle de création dans une LS).

Louvel nous dit partir d'un constat. Celui d'une «longue inféodation de l'image au texte ${ }^{52} \gg$. Certains critiques, nous dit-elle encore, ont envisagé ce rapport en y voyant un impérialisme. Déjà, l'entrée en résonance est aiguë. Dans le contexte des Deaf Studies, là d'où je parle, la prégnance de l'idée de cette inféodation de l'image au texte dans l'histoire des idées a une importance cruciale. En effet, rappelons-nous que c'est en raison de leur iconicité que les LS ont été discréditées — « grossly material images », disait l'abbé Terra. La mémoire, la conscience de cette subordination hante toute la réflexion théorique à propos de la création en LS. C'est elle qui laisse, transversalement, les adhérences les plus récalcitrantes à dissoudre.

La méthodologie que Louvel entend concevoir dans cet ouvrage aspire à l'essor d'une critique intermédiale des œuvres laissant à chaque art, littérature et peinture, son espace de liberté, délivrée de cette obligatoire hiérarchisation, de cette tentation de la sujétion de l'une par l'autre :

so Liliane Louvel, Le tiers pictural: pour une critique intermédiale, Rennes, Presses universitaires de Rennes, 2 oro.

si Ibid., p. 8.

${ }^{2}$ Ibid., p. 7. 
le rapport texte/image a été conçu en termes agonistiques, en termes de hiérarchie, de domination. Comme si l'on n'avait pu s'empêcher de le penser comme un rapport forcément vertical, comme une lutte de territoires. On pourrait proposer, pour suivre l'exemple de Deleuze, en d'autres domaines, de plutôt l'appréhender en termes de plateau, plateau situé entre texte et image, qui se développeraient horizontalement à la manière du rhizomes3.

Dans le cas qui m'occupe, c'est une pyramide qui met à son sommet l'attention à la seule dimension linguistique qu'il s'agit de défaire pour la mettre en relation dynamique et d'égale valeur avec les autres composantes esthétiques des œuvres : corps en mouvement, image, espace. Dans la méthodologie que je m’attache à développer, il y a des pôles, entre lesquels s'exerce une force d'attraction qui les met en mouvement; des sphères plutôt que des plateaux; du magnétisme plutôt que de la racine. Cependant, les principes qui guident la critique intermédiale que propose Louvel sont d'une formidable inspiration pour la constitution d'une critique pour les œuvres en LS. Comment appréhender la relation entre ces différents pôles lorsqu'elle passe de la performance à sa réception?

Ce que Louvel nomme «tiers pictural » conceptualise un événement de lecture. La locution désigne le phénomène qui se manifeste chez le lecteur lorsqu'il entre en contact avec l'évocation plus ou moins explicite de l'image dans le texte, fluctuation continuelle entre les deux versants de la dyade. Le tiers pictural signifie la manière dont l'image surgissant du texte affecte le lecteur.

Comment saisir le phénomène équivalent qui surviendrait lors de l'expérience de réception d'une œuvre en LS ? Si on appréhende l'œuvre en cultivant la curiosité de la configuration mouvante de l'ensemble de ses pôles, peut-on imaginer voir survenir un tiers qui soit synesthète, c'est-à-dire une suite de moments entrelacés où s'éprouve le chevauchement, toujours différemment agencé, des composantes esthétiques de l'œuvre?

Pour parvenir à traduire de manière à aménager des espaces d'accueil pour ces œuvres, pour parvenir à traduire contre la violence, c'est l'ensemble de ces composantes esthétiques qu'il nous faut pouvoir faire tenir dans le langage. Une démarche critique inspirée par les agencements de percepts, d'expériences somaesthésiques, de concepts qu'elle cherche à capter apparaît comme une voie prometteuse. Dans sa version appliquée, elle sera utile pour nourrir la démarche de traduction des œuvres. Peut-être même pourrions-nous trouver judicieux de nous aventurer hors des territoires réservés

\footnotetext{
53 Ibid., p. 20.
} 
aux langues écrites - l'encre sur le papier - et proposer des dispositifs de traduction qui soient aussi intermédiaux, indisciplinés en somme.

\section{CONCLUSION}

Au fil de ce texte, j'ai tenté de mettre en évidence l'incontournable lien entre art et politique qui traverse la pensée portant sur la création issue des LS. L'asymétrie des rapports qui structurent les relations entre les signants et les parlants possède une histoire aussi longue que celle de la pensée concernant les rapports entre le corps et le langage. Des idéologies s'y sont formées, s'y sont opposées et, entre elles, existent des rapports de pouvoir. Cette asymétrie, selon la plupart des auteurs cités dans cet article, porte les marques d'un rapport colonial qui se manifeste, entre autres, sur le plan légal et institutionnel, avec des effets concrets sur la vie des individus.

De façon plus diffuse, cependant, ces idéologies qui se matérialisent dans le cadre de mesures, de politiques, de lois, ou plus prosaïquement de logistique, forment la fréquence basse de l'imaginaire commun et fondent les représentations les plus passives des LS. Or, la création porte en puissance la faculté de les transformer, non seulement par son propos lorsqu'elle formule un commentaire, mais aussi par son travail sur les formes. Avec elle, la critique des œuvres et le développement de méthodologies, comme on ajuste une focale, travaillent à rebours les représentations instituées. Il s'agit, en somme, de territoires, d'espaces habités. Lorsque la création qui en est issue apparaît sur les scènes des théâtres ou des festivals où on ne la voit pas d'ordinaire, les LS prennent place. Lorsqu'une critique d'œuvre ou une analyse théorique portant sur la création en LS s'achemine jusque dans les lieux dédiés au discours sur les arts, un espace s'ouvre. Par cette pratique est reconfiguré le tracé des lignes du partage du sensible, qui commande non seulement les inclusions et les exclusions, mais fabrique aussi la fibre du commun. 


\title{
Le tiers synesthète : espace d'accueil pour la création en langue des signes
}

\author{
Julie Chateauvert, Université Paris 8
}

\section{RÉSUMÉ}

Le présent texte s'interroge sur l'espace cognitif à ouvrir afin de rendre possible, au sein de la théorie littéraire et artistique, une réception entière des œuvres de création en langues des signes. Il propose le développement d'une théorie de l'œuvre et de la réception comme geste politique nous rendant apte à répondre à l'invitation de Rada Iveković pour parvenir à traduire contre la violence.

\section{ABSTRACT}

With this text, I would like to reflect on the opening up of a cognitive space that would allow full acceptance, within literary and artistic theory, of narrative works created in Sign Languages. The text proposes the development of a descriptive theory of the work of art and its reception as a political gesture, which would enable us to respond to Rada Iveković's invitation to translate against violence.

\section{NOTE BIOGRAPHIQUE}

Julie Chateauvert est chercheuse postdoctorale à l'Université Paris 8 où elle est affiliée à l'unité de recherche Transferts critiques et dynamiques des savoirs. Elle est la rédactrice de l'entrée Poetry dans The SAGE Deaf Studies Encyclopedia, publiée chez SAGE en 2016. Elle a également publié l'article «Qu'est-ce que la littérature en langue des signes? » dans le numéro 149 de la revue de linguistique L'information grammaticale. Son travail est nourri des 20 ans passés à agir comme compagne de route auprès des communautés sourdes du Québec et de la France qu'elle tient à reconnaître et à remercier chaleureusement. 